\title{
Elemental Mapping Tomography with Surrogate Alignment
}

\author{
Shixin Wang \\ Micron Technology, Inc., 8000 S. Federal Way, Boise, ID 83707
}

In a transmission electron microscopy (TEM) system, various techniques can be used to obtain twodimensional chemical maps. Two commonly used signal sources for $2 \mathrm{D}$ elemental mapping are x-ray energy dispersive spectroscopy (EDS) and electron energy loss spectroscopy (EELS). The first method extracts elemental specific maps through a synchronized EDS and scanning transmission electron microscopy (STEM) imaging. The second mapping method can be performed through either energy filtered transmission electron microscopy (EFTEM) imaging or extraction from synchronized spectra and STEM. Using tomographic calculation, it is possible to reconstruct a chemical-specific 3D volume from a series of maps taken from different tilt angles [1,2]. Tomography reconstruction based on elemental maps often runs into the difficulty of low signal intensity, which is common with most elemental mapping methods. The low intensity often results in poorly aligned elemental maps within a tilt series. This poor alignment subsequently leads to a poor or misleading result in reconstructed tomograms.

The direct solution for increasing the intensity of the elemental maps is to prolong the collection time. High intensity EFTEM mapping using accumulation and drift correction has been demonstrated [3]. However, this approach is often time consuming and not suitable to a large number of maps for tomography reconstruction. One alternative method for overcoming the difficulty of low intensity is to use a surrogate alignment. Ideally, a surrogate alignment is obtained from an image series taken at the same time as the maps being studied. The image used for surrogate alignment should have the following properties: (1) high intensity, (2) good representation of spatial definition of the elemental map, and (3) negligible stage drift relative to the map. In EFTEM imaging, the zero-loss image, or a low-loss image, is a good candidate for the elemental maps. In EDS mapping or EELS spectrum imaging, the STEM image is a natural choice for the surrogate alignment images.

In this work, we demonstrate the method using a series of EFTEM Ti map and zero-loss image pairs. Figure 1A shows a Ti map obtained from Ti core-loss signal. Figure 1B is the zero-loss image taken immediately after the Ti map. A Ti map and a zero-loss image were taken at each stage tilt, after a sufficient drift settling time. The tomography program package IMOD [4] was used on image alignment, tomogram reconstruction, and visualization. In general, the zero-loss images have a much higher intensity and richer spatial information than the Ti maps. Subsequently, the alignment data obtained from the zeroloss image gives more precise spatial alignment within the tilt series. When the drift with Ti maps is negligible, the zero-loss images can be used as reference images to obtain high quality alignment data, which we may call the surrogate alignment. In this study, the alignment obtained through zero-loss images was directly applied to the Ti map series in order to align the data set. The reconstructed 3D Ti maps are shown in Figure 2. This result shows the validity of the surrogate alignment procedure. This Procedure can be applied to low signal tomography in general.

\section{References:}

[1] P. A. Midgley, and M. Weyland, "3D electron microscopy in the physical sciences: the development of Z-contrast and EFTEM tomography", Ultramicroscopy 96 (2003) 413-431. 
[2] Q. Jin (2010). An application of energy-filtered elemental mapping tomography in semiconductor device analysis. Microscopy and Microanalysis, 16(Suppl. 2), pp 1922-1923. [3] S. X. Wang (2010). High sensitivity EFTEM elemental mapping with unlimited acquisition time. Microscopy and Microanalysis, 16(Suppl. 2), pp 1536-1537.

[4] J.R. Kremer, D.N. Mastronarde, and J.R. McIntosh, "Computer visualization of threedimensional image data using IMOD”. J. Struct. Biol. 116, (1996) 71 -76.

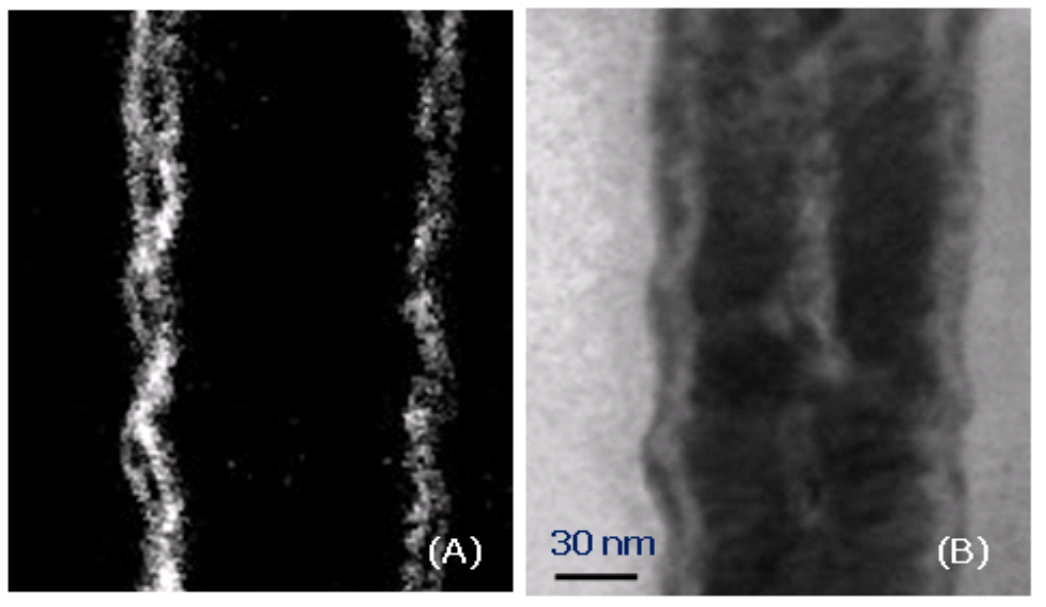

Figure 1. (A) Ti map from energy filtered imaging. (B) Zero-loss image taken immediately after the Ti map.

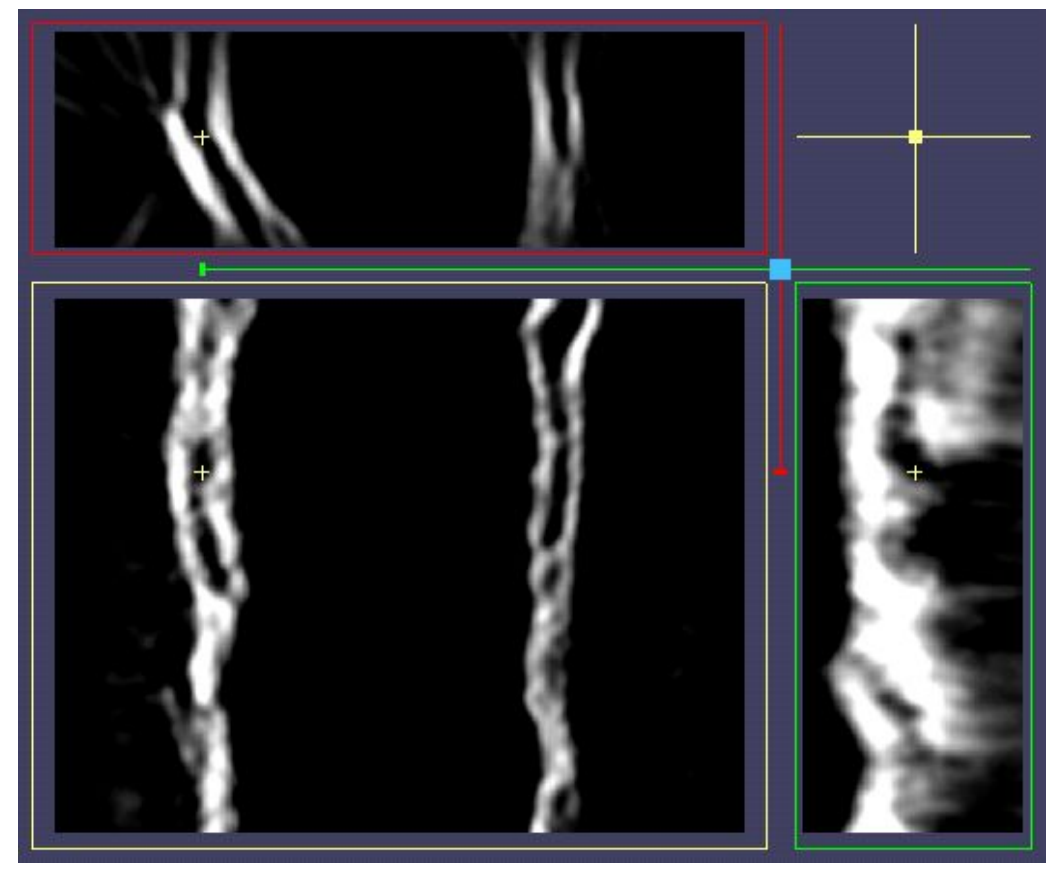

Figure 2. Reconstructed tomogram as viewed in three orthogonal sections. 